\title{
Correction of prominent ear by the two parallel incision technique with definition and folding of the antihelix by vertical mattress sutures
} Ashraf Khaled, Ramez Reda, Ahmed Kamel, Samir Shokr A. Rhman

Department of Otolaryngology, Faculty of Medicine, Beni Suef University, Beni Suef, Egypt

Correspondence to Ramez Reda, MD, Department of Otolaryngology, Faculty of Medicine, Beni Suef University, Beni Suef, Egypt. Tel: 00201225012157, 00202/22315648; e-mail: drramez2003@yahoo.com

Received 10 August 2018

Accepted 10 August 2018

The Egyptian Journal of Otolaryngology 2018, 34:217-223

\begin{abstract}
Background incision and vertical mattress sutures to define antihelix.

\section{Patients and methods} antihelix with vertical mattress sutures and ellipse of skin was removed.

Results had keloid.

Conclusion rates.

Keywords:

antihelix, cartilage incision, prominent ear, vertical mattress suture

Egypt J Otolaryngol 34:217-223

(1) 2018 The Egyptian Journal of Otolaryngology

$1012-5574$
\end{abstract}

Multiple techniques have been advocated over the years for correction of the prominent ear. The basic techniques used to correct prominent ears with a missing antihelix depends on incision, scoring, bending and reposition of the auricular cartilages. This study was undertaken to correct prominent ears using cartilage

This study was carried out on 13 patients (25 ears) in the period between February 2014 and June 2017, twelve patients of them were presented with bilateral prominent ears and one patient was presented with unilateral prominent (bat) ear. This series comprised 8 males and 5 females. Their ages ranged between 7 to 33 years. In this technique 2 parallel incision in auricular cartilage, definition of

Satisfactory results were obtained in all patients. Complications occurred in 2 patients only: one of them had mild protrusion of upper third of auricle and the other

Our technique produces natural-looking of the operated ears with low complication

\section{Introduction}

Aside from the excision of auricular tags and the repair of traumatically clefted ear lobes, the prominent ear is the most commonly treated external ear deformity [1].

Lack of formation of the antihelix between the 12th and the 16th week of embryonic life results in this protrusion [2].

Children with prominent ears are often patient for verbal and physical abuse, which affects them psychologically and socially. Children's parents have become alert about all these problems. Hence, otoplasty has both esthetic and functional purposes [3].

Haytoglu et al. [4] mentioned in 2015 that several techniques can give satisfactory results for correction of prominent ear, but few deal with all its components.

Normally, the auriculocephalic angle is between 25 and $30^{\circ}$. If it is more than $40^{\circ}$, this can be considered abnormal. Moreover, the normal conchoscaphal angle is $\sim 90^{\circ}$. If it is more obtuse, it will require surgery $[5,6]$.
Most of the techniques used to correct prominent ears with a missing antihelix are based on incision, scoring, bending or reposition of the auricular cartilaginous framework [7].

Many techniques for correction of prominent ear have been described in the literature, with satisfactory results being achieved by many of them. In spite of this, complications may occur, such as infection or poor scar formation (keloids and the hypertrophic scarring) [5].

Anesti and Moss [8] reported that the multitude of different approaches indicates that there is no clearly definitive technique for correcting prominent ears.

\section{Patients and methods}

This study was carried out on 13 patients (25 ears). Twelve of them presented with bilateral prominent ears,

This is an open access journal, and articles are distributed under the terms of the Creative Commons Attribution-NonCommercial-ShareAlike 4.0 License, which allows others to remix, tweak, and build upon the work non-commercially, as long as appropriate credit is given and the new creations are licensed under the identical terms. 
and one patient presented with unilateral prominent (bat) ear. Cases were assessed and operated by the technique described below. The surgeries were performed between February 2014 and June 2017 by the ENT Department of Beni Suef University. The operated patients were between 7 and 33 years of age (average of 14 years old) and included five female patients and eight male patients. The study was approved by the ethics committee of Beni Suef University. A written informed consent was obtained from all patients for surgery and publishing their photos.

Each case was subjected preoperatively to full ENT examination, especially the auricles, external auditory canals and tympanic membranes to exclude any other congenital anomalies that may interfere with the surgical correction of the auricles. The examination was focused on the auricle, especially the development of the antihelical fold and its crura, and the stiffness of auricular cartilage. Preoperative photography was carried out from the front, back and both sides. The same photos were taken 3 months postoperatively.

All operations were performed under general anesthesia. Patients were assessed and discharged on the first postoperative day.

\section{Surgical technique}

(1) Surgery was performed under general anesthesia.

(2) The patient was in the supine position with the first ear to be operated facing upward.

(3) Infiltration of the skin with $1: 200000$ epinephrine solution was then carried out.

(4) Marking of the site and shape of the new antihelix was carried out by introducing insulin needles on the anterior surface of the ear (Figs 1 and 2). These anterior markings refer to the site of cartilaginous incision.

Fig. 1

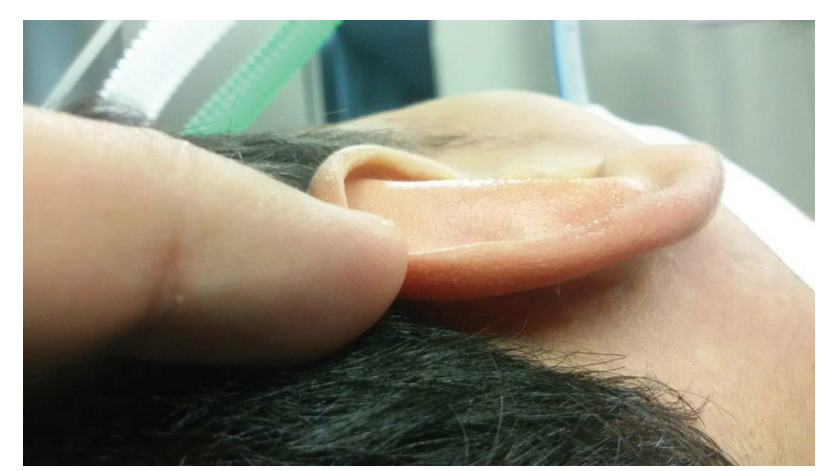

Marking of the antihelix (right ear).
(5) Posterior markings were carried out by scalpel no. 15 to determine the site of skin island that would be resected (Fig. 3).

(6) Elliptical incision and resection of the posterior skin island was carried out according to the previous markings, so that the resulting scar was positioned in the retroauricular crease.

(7) Posterior dissection of the ear in the subperichondrial plane was carried out to expose the cartilage.

(8) For incision of the cartilage, two slightly oblique parallel incisions to the helix were made $1-2 \mathrm{~mm}$ in width, starting from the triangular fossa and extending to the root of the helix. Presence of this cartilaginous strip will prevent any sharp angle at the antihelix.

(9) Definition and folding of the antihelix with vertical mattress sutures using 4.0 vicryl suture from perichondrium to perichondrium was performed (Figs 4 and 5).

(10) Skin closure with the simple suture technique using 4.0 silk suture was then performed.

(11) After that, suturing of the skin of the posterior surface of the auricle to skin over the mastoid was carried out, in order to fix the auricle at its new position and to avoid traction on the skin sutures.

(12) The same technique was used in the other ear in bilateral cases.

(13) Dressing with pieces of cotton filling the concavities of the ear, then putting gauze on it was then carried out. We did not find the need to use crepe bandage around the head at the end of the operation.

Fig. 2

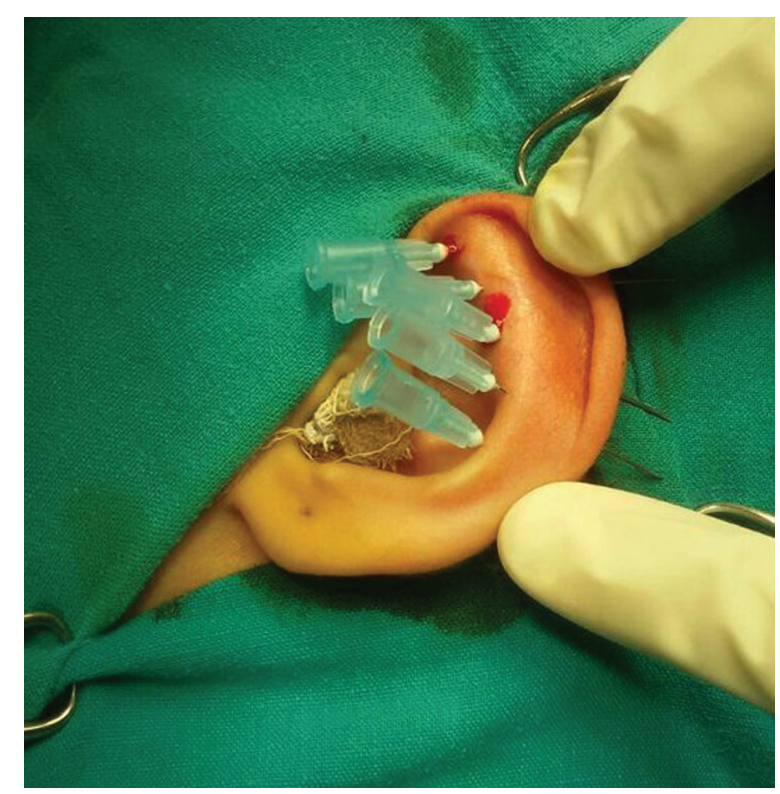

Introducing insulin needles to determine antihelix (left ear). 
Fig. 3

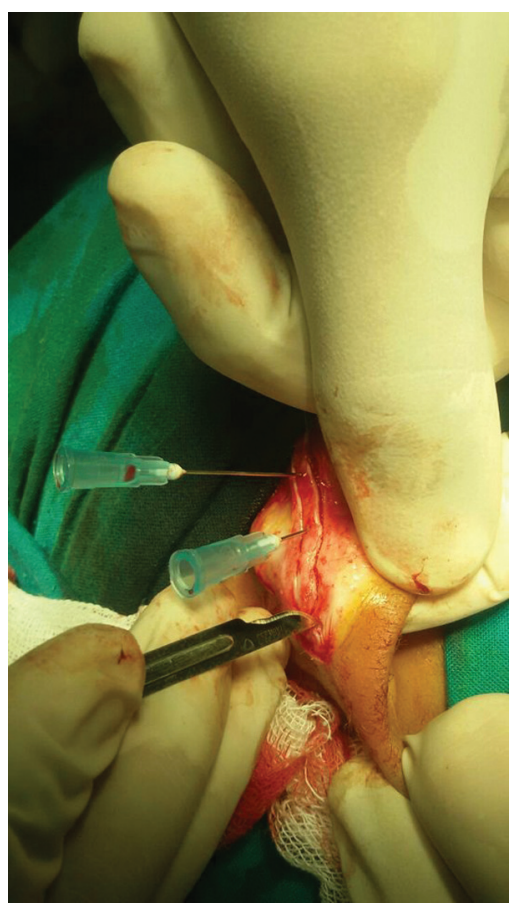

Elliptical incision of the skin (right ear).

Fig. 5

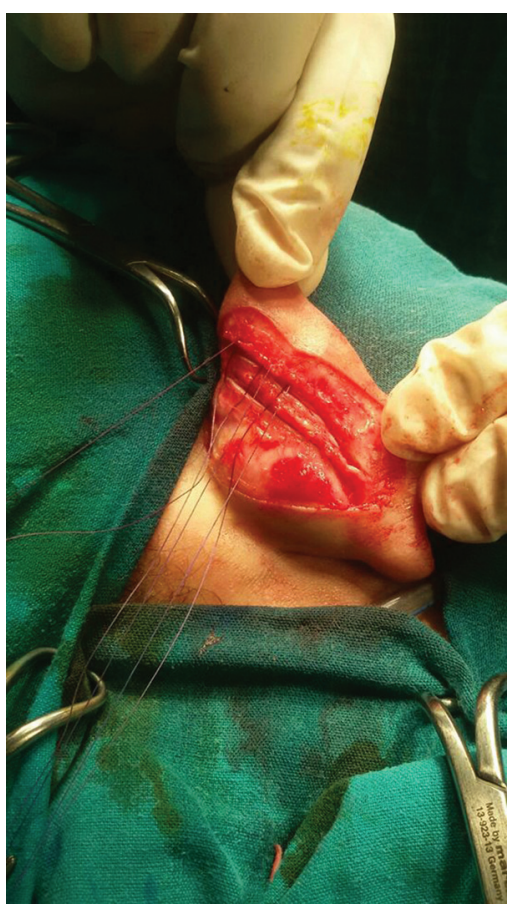

Suturing and folding of antihelix (right ear).

(14) Antibiotic therapy was maintained for 7 days in addition to analgesic anti-inflammatory drugs.

(15) Postoperative follow-up consultations were held 1, 2 weeks, 1 month, and every 3 months after the procedure. The sutures were removed 1 week postoperatively.
Fig. 4

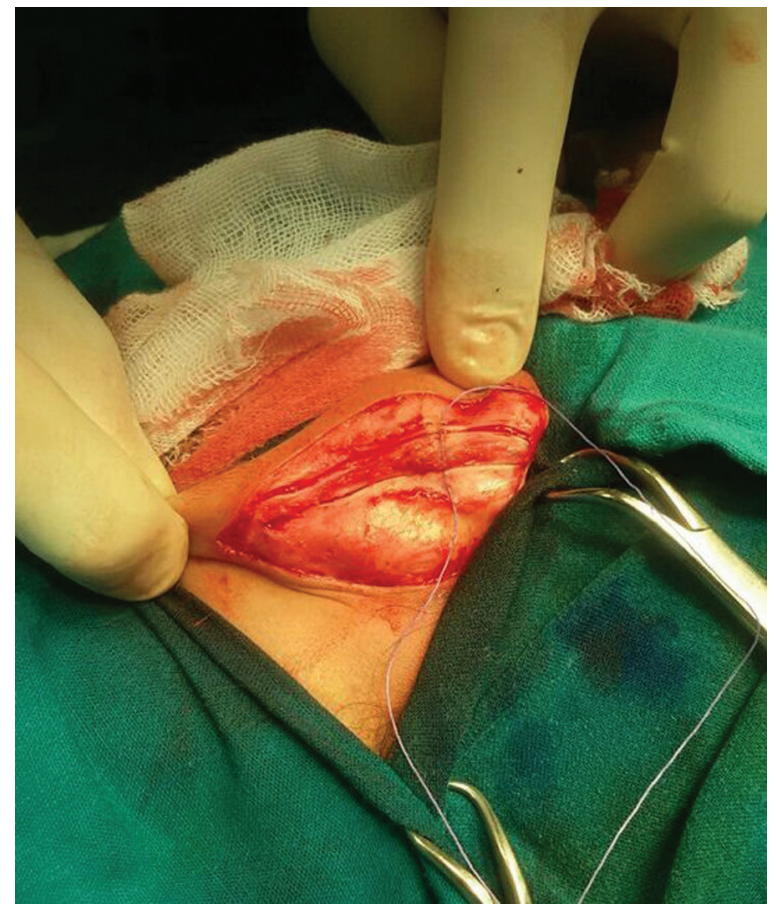

Incision and folding of the cartilage (left ear).

Fig. 6

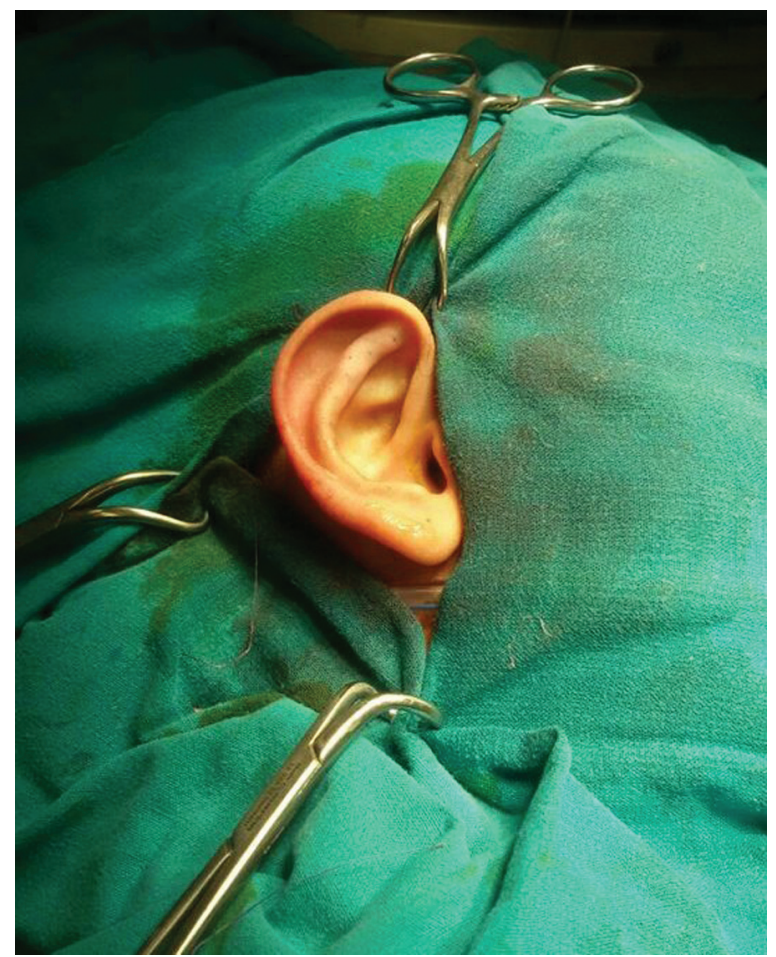

The corrected right ear with the newly formed antihelix.

\section{Results}

A total of 13 patients (25 ears) underwent correction of prominent ears by the technique presented in this study. The technique used was cartilage incision 
Fig. 7

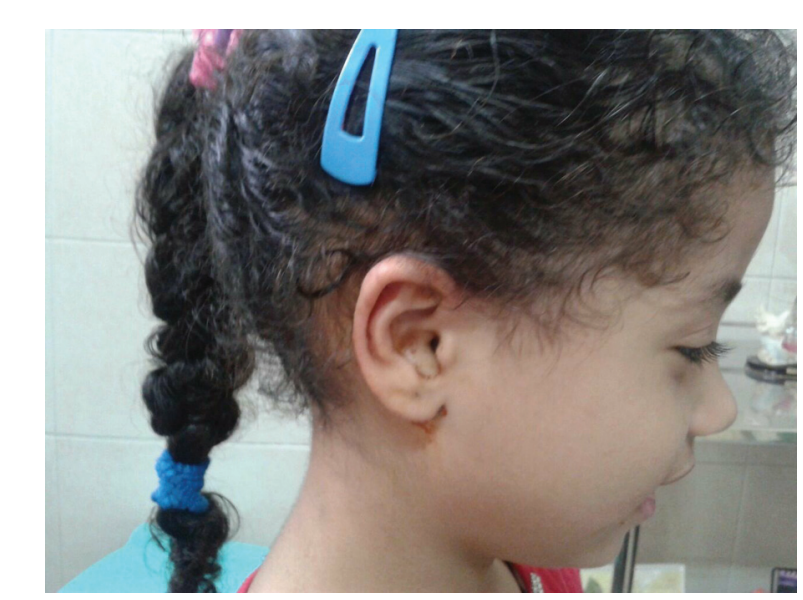

A corrected right ear 3 months postoperatively.
Fig. 8

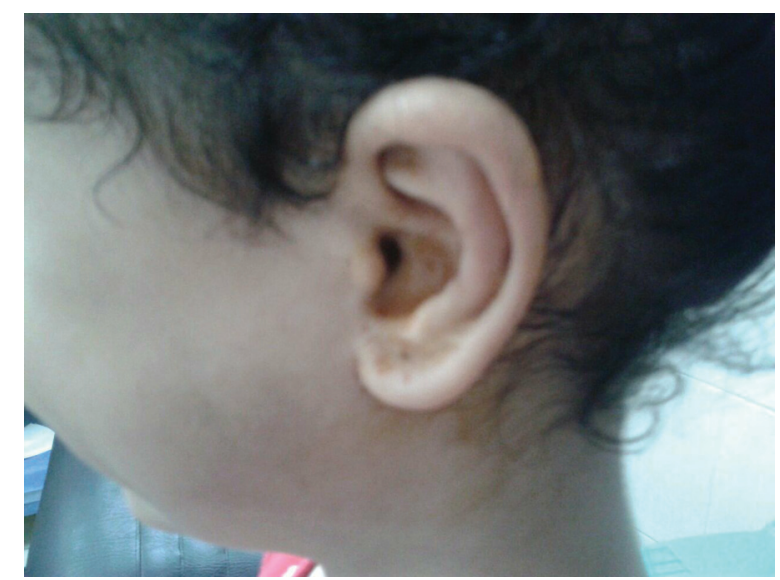

A corrected left ear 3 months postoperatively.

Fig. 9

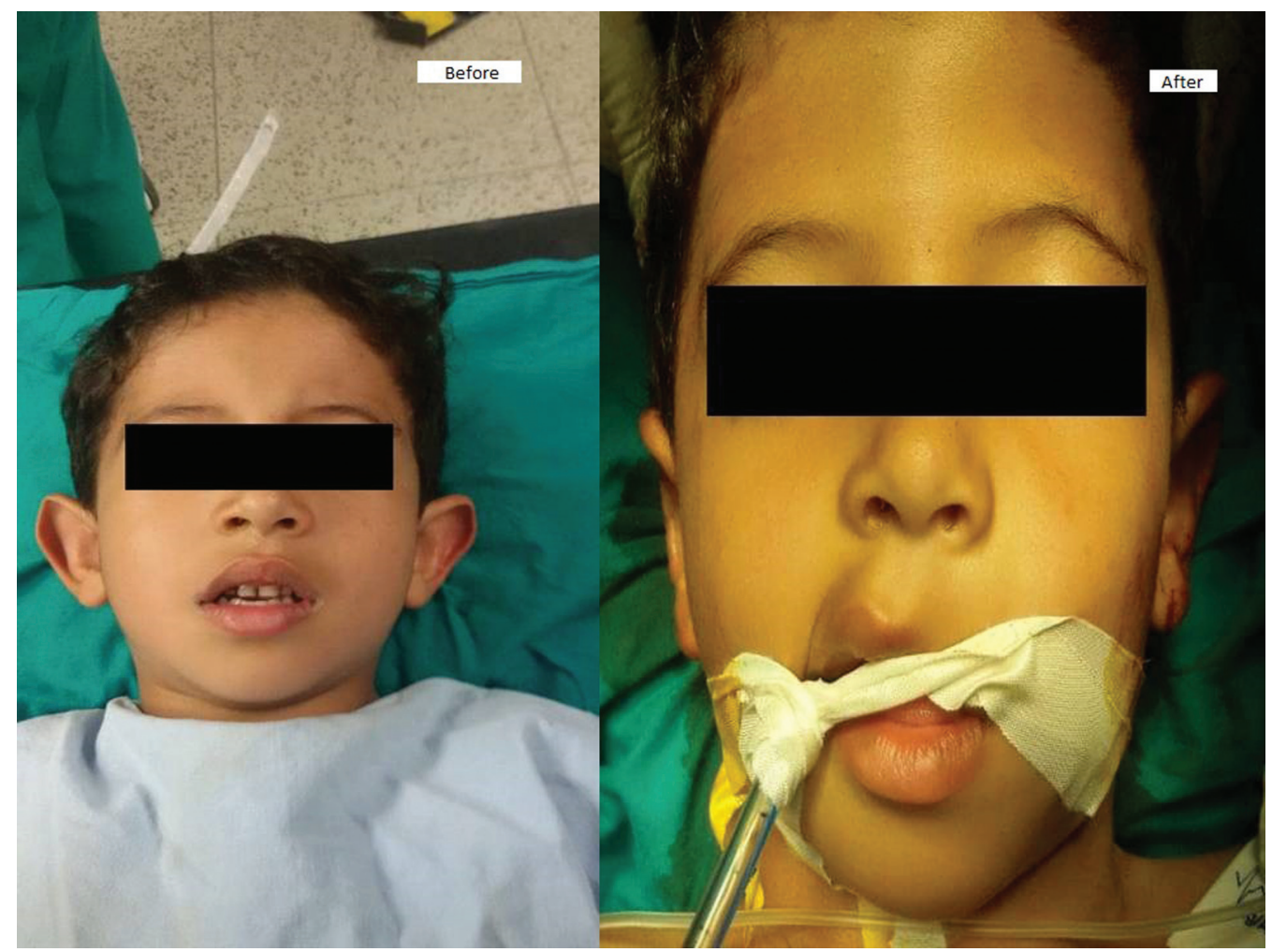

One of the patients: before beginning the surgical technique and at the end of surgery (front view).

followed by vertical mattress sutures for antihelix definition.

The postoperative results were satisfactory for the patients and the surgeons. Complications were encountered only in two cases. One of them suffered suture keloids and the other case has mild protrusion of the upper third of the auricle. No postoperative hematoma, wound or cartilage infection nor scar problems were encountered in our study (Fig. 6-11).

\section{Discussion}

Many techniques have been used over the previous years for correction of the prominent ear. They included excision, bending, scoring or reposition of the auricular cartilage. The multiplicity of approaches indicate that there is no single technique that can recreate the complex three dimensional nature of the normal human ear [8-10]. 
Fig. 10

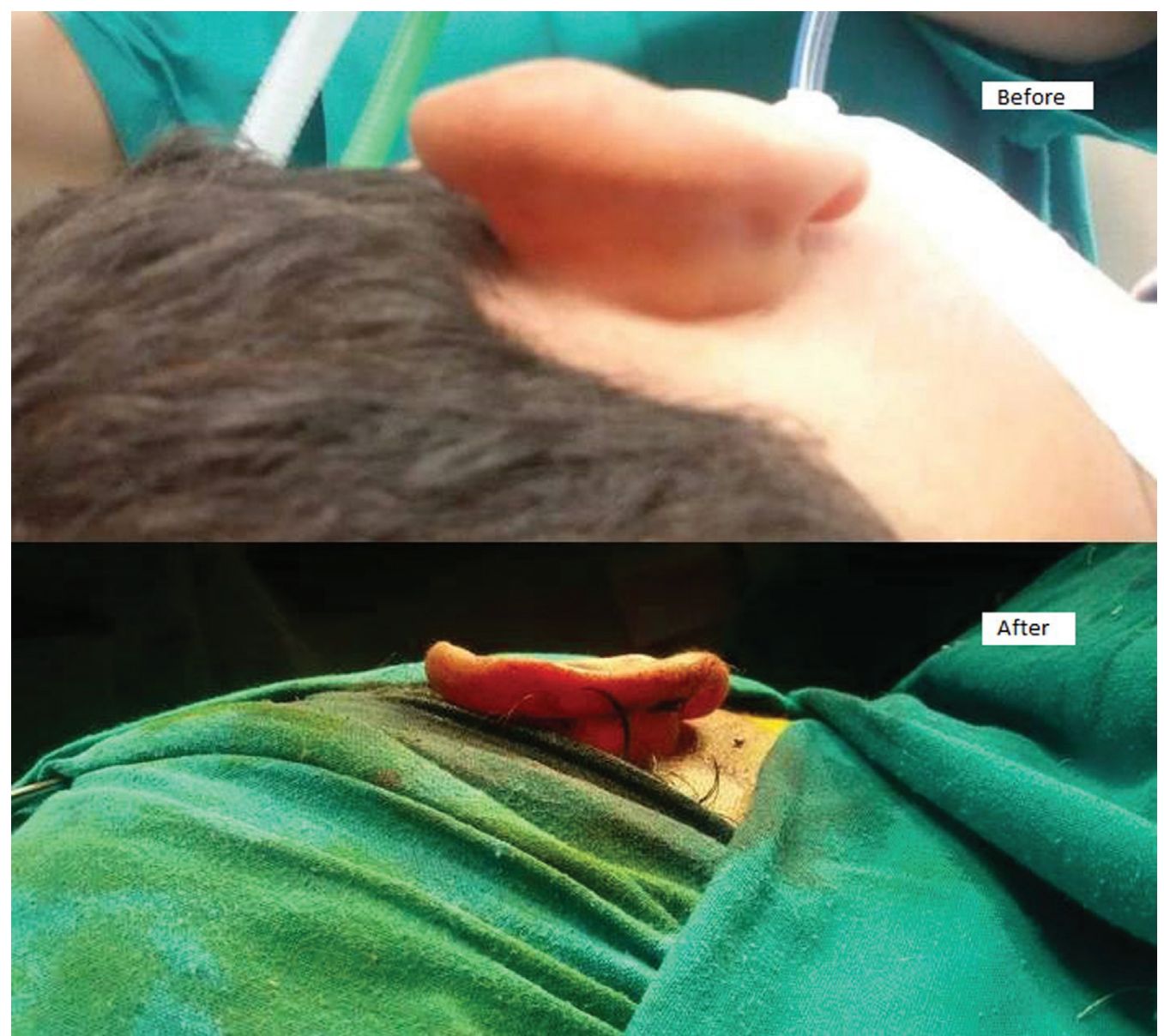

One of the patients: before beginning the surgical technique and at the end of surgery (lateral view).

Dieffenbach [11] was the first one to describe a technique for surgical correction of prominent ears in 1845 . Since that time, many techniques have been developed, but the goals of pinnaplasty have the same issue, as mentioned by McDowell [12]. The protrusion of the upper part of the ear should be decreased, and the helix should be more lateral than the antihelix, when seen from the front. Moreover, the helix should be smooth and regular. The postauricular sulcus should be of average size. The operated ear should not be placed too close to the head, and the contours and positions of both ears should match closely, even if not symmetrical.

In this study, the age of our patients ranged between 7 and 33 years. In our series, the operative time for otoplasty ranged between 1 and $2 \mathrm{~h}$ for bilateral cases, which implies that this technique is not time consuming.

Patients were followed-up in our study for a period that ranged between 3 to 24 months, which is considered an acceptable period for technique evaluation. Follow-up period ranging between 6 months to 2 years was reported by other authors [13].
Numerous techniques and modifications have been tried for solving a prominent ear deformity. Their basic concept originates from Mustard's suture. These are the scoring technique described by Chongchet and Stenstrom in addition to the combined suture and scoring technique described by converse. Techniques for cavum rotation and lobule plasty have also been presented [14].

After using several techniques, in 1963, Mustarde [15], proposed avoiding making excisions, incisions or weakening the cartilage, because he considered that a simple suture was sufficient to maintain the shape of the ear during healing.

In our opinion, the cartilaginous incision provides a suitable break of the cartilaginous spring in patients, especially in patients with thick cartilages.

For definition of the antihelix, two to four sutures were used, as needed in every case. Excessive tightening of the sutures was avoided to prevent esthetic impairment. 
Fig. 11

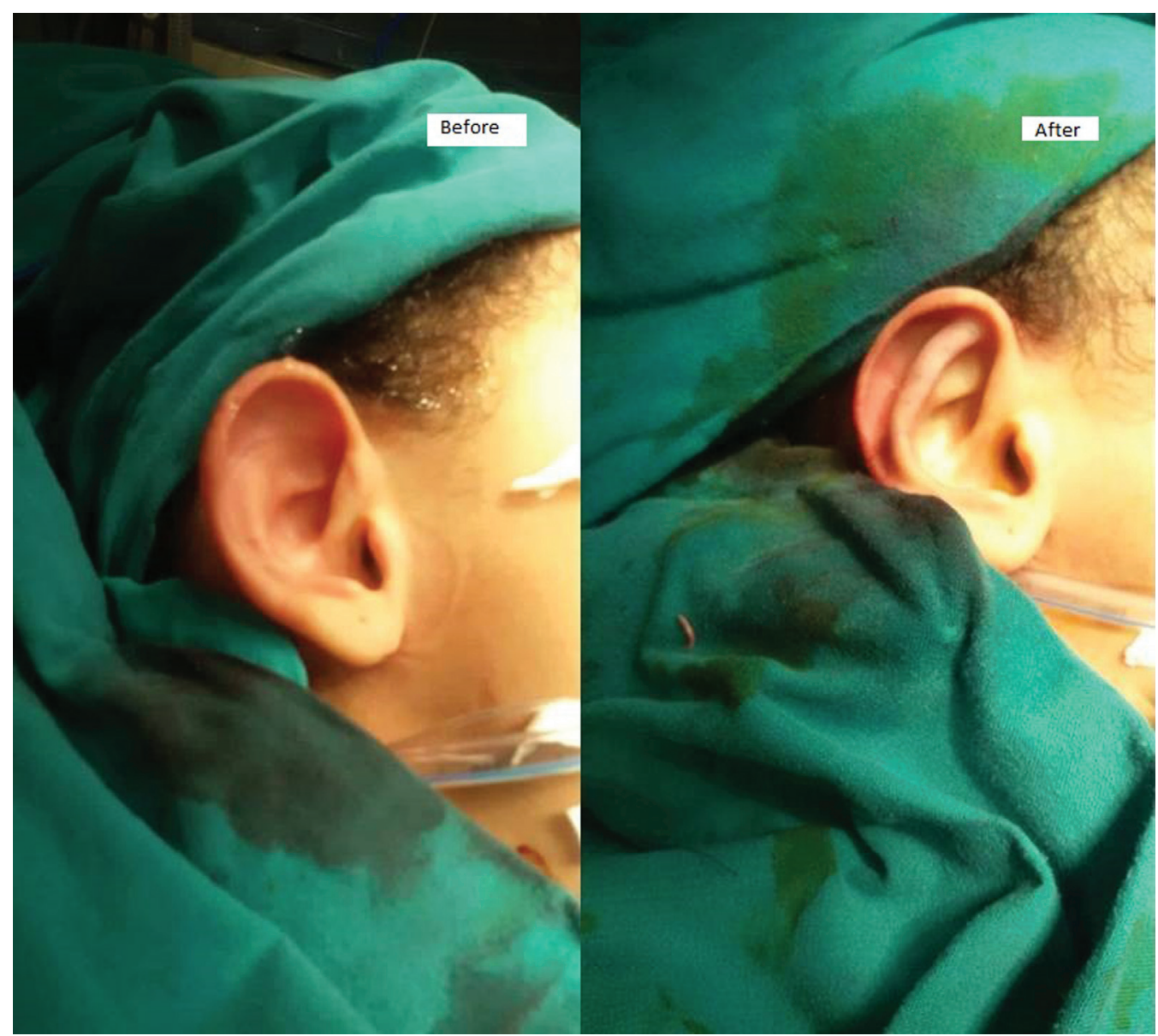

One of the patients: before beginning the surgical technique and at the end of surgery showing the newly formed antihelix (lateral view).

Adamson et al. [16] mentioned that cartilage-cutting procedures have higher rates of revision and persistent postoperative stigmata than cartilage-sparing procedures.

Our procedure consists of cartilage incision and sparing, and we agree with the findings of Adamson and colleagues as regards the postoperative results and revision rates.

Many complications of otoplasty were recorded. It included hematoma, infection, chondritis, and, maybe, necrosis. Late complications included scarring, patient dissatisfaction, suture problems, and dysesthesias.

The only complications that we encountered were a suture keloid in one case and mild protrusion of the upper third of the auricle in the other case. We did not encounter any instances of hematomas, skin necrosis, suture extrusion and chondritis.

\section{Conclusion}

The best treatment of prominent ears is achieved by a simple technique with sparing of the cartilage and definition of the antihelix by vertical mattress sutures. The technique used in this study produces a natural-look of the operated ears with low complication rates.

\section{Financial support and sponsorship \\ Nil.}

\section{Conflicts of interest}

There are no conflicts of interest.

\section{References}

1 Posnick J. Aesthetic alteration of prominent ears: evaluation and surgery. In: Principles and practice of orthognathic surgery, Saunders, 2014; Chapter 39:1703-1745.

2 Azuara E. Aesthetic otoplasty with remodeling of the antihelix for the correction of the prominent ear. Arch Facial Plast Surg 2000; 2:57-61.

3 Purcell E, O'Neill A, Regan P. A different type of 'glue ear': report of an unusual case of prominent ears. Ear Nose Throat 2003; 82:702-703.

4 Haytoglu S, Haytoglu T, Muluk N, Kuran G, Arikan O. Comparison of two incisionless otoplasty techniques for prominent ears in children. Int $\mathrm{J}$ Pediatr Otorhinolaryngol 2015; 79:504-510.

5 Firmin F, Sanger C, O? toole G. Ear reconstruction following severe complication of otoplasty. J Plast Reconstr Aesthet Surg 2008; 61:513-520.

6 Handler E, Song T, Shih C. Complications of otoplasty. Facial Plast Surg Clin North Am 2013; 21:653-662. 
7 Nolst G. Otoplasty: a modified anterior scoring technique. Facial Plast Surg 2004; 20:277-285.

8 Anesti K, Moss A. Otoplasty morbidity. Mod Plast Surg 2013; 1:28-33.

9 Janis J, Rohrich R, Gutowski K. Otoplasty. Plast Reconstr Surg 2005; 115:60e-72e

10 Campbell A. Otoplasty. Facial Plast Surg 2005; 21:310-316.

11 Dieffenbach J. Otoplasty training. the operative surgery. Leipzig: Brockhaus; 1848. 395.

12 McDowell A. Goals in otoplasty for protruding ears. Plast Reconstr Surg 1968; 41:17-27.
13 Chao B, Chung H, Park J. Surgical correction of prominent ear using modified tube technique and posterior approach. J Craniofac Surg 2003; 14:767-773.

14 Zayed E, Hegazy H. Correction of prominenant ear by the use of y-shaped cartilage strip, assisted conchal reduction technique. Egypt $\mathrm{J}$ Plast Reconstr Surg 2007; 31:139-148.

15 Mustarde J. The correction of prominent ears using simple mattress sutures. Br J Plast Surg 1963; 16:170-178.

16 Adamson P, McGraw B, Tropper G. Otoplasty: critical review of clinical results. Laryngoscope $1991 ; 101: 883-888$. 